\title{
Pion valence quark distribution from current-current correlation in lattice QCD
}

\author{
Raza Sabbir Sufian $\odot,{ }^{1}$ Colin Egerer, ${ }^{2}$ Joseph Karpie, ${ }^{3}$ Robert G. Edwards $\odot,{ }^{1}$ Bálint Joó, ${ }^{1}$ Yan-Qing Ma, ${ }^{4,5,6}$ \\ Kostas Orginos, ${ }^{1,2}$ Jian-Wei Qiu, ${ }^{1}$ and David G. Richards ${ }^{1}$ \\ ${ }^{1}$ Thomas Jefferson National Accelerator Facility, Newport News, Virginia 23606, USA \\ ${ }^{2}$ Physics Department, William and Mary, Williamsburg, Virginia 23187, USA \\ ${ }^{3}$ Physics Department, Columbia University, New York City, New York 10027, USA \\ ${ }^{4}$ School of Physics and State Key Laboratory of Nuclear Physics and Technology, \\ Peking University, Beijing 100871, China \\ ${ }^{5}$ Center for High Energy physics, Peking University, Beijing 100871, China \\ ${ }^{6}$ Collaborative Innovation Center of Quantum Matter, Beijing 100871, China
}

(Received 22 January 2020; accepted 31 August 2020; published 15 September 2020)

\begin{abstract}
We extract the pion valence quark distribution $q_{\mathrm{v}}^{\pi}(x)$ from lattice QCD (LQCD) calculated matrix elements of spacelike correlations of one vector and one axial vector current analyzed in terms of QCD collinear factorization, using a new short-distance matching coefficient calculated to one-loop accuracy. We derive the Ioffe time distribution of the two-current correlations in the physical limit by investigating the finite lattice spacing, volume, quark mass, and higher-twist dependencies in a simultaneous fit of matrix elements computed on four gauge ensembles. We find remarkable consistency between our extracted $q_{\mathrm{v}}^{\pi}(x)$ and that obtained from experimental data across the entire $x$ range. Further, we demonstrate that the oneloop matching coefficient relating the LQCD matrix computed in position space to the $q_{\mathrm{v}}^{\pi}(x)$ in momentum space has well-controlled behavior with Ioffe time. This justifies that LQCD-calculated current-current correlations are good observables for extracting partonic structures by using QCD factorization, which complements to the global effort to extract partonic structure from experimental data.
\end{abstract}

DOI: $10.1103 /$ PhysRevD.102.054508

\section{INTRODUCTION}

The pion, being both a Nambu-Goldstone boson and the lightest bound state in quantum chromodynamics (QCD), highlights the challenges in creating consistent theoretical and phenomenological frameworks to describe its partonic structure. The shape of the pion valence parton distribution functions (PDFs) extracted from experimental data [1-5] in different analyses [6-12] are in sharp contrast among themselves and with perturbative QCD (pQCD)-based frameworks $[13,14]$ at large longitudinal momentum fractions $x$. Central to the disparity is whether the pion PDF has a softer (harder) $(1-x)^{2}[(1-x)]$ falloff as $x \rightarrow 1-$ various model calculations [15-21] exemplify this contrast.

The limited available phase space for partonic interactions at large $x$ localizes quantum fluctuations such that large- $x$ dynamics is constrained by confinement, in effect increasing parton correlations as $x \rightarrow 1$. As the quark

Published by the American Physical Society under the terms of the Creative Commons Attribution 4.0 International license. Further distribution of this work must maintain attribution to the author(s) and the published article's title, journal citation, and DOI. Funded by SCOAP . distribution at large $x$ is sensitive to nonperturbative quark-gluon dressing, a description of its behavior will also elucidate our understanding of the generation of mass in QCD through dynamical chiral symmetry breaking. Unraveling the complexities of the valence and sea quark contents of the pion is spearheaded by several upcoming experiments-Jefferson Lab tagged deepinelastic scattering experiments [22], Drell-Yan measurements at the COMPASS experiment [23] and, also the future Electron-Ion Collider facility [24]. A first-principles lattice QCD (LQCD) determination of the pion valence PDF $q_{\mathrm{v}}^{\pi}(x)$ with controlled statistical and systematic uncertainties is particularly well timed and solicits a synergy of increasing importance between experimental and theoretical efforts.

PDFs are not direct physical observables, such as cross sections, due to the QCD color confinement. Experimental extraction of $x$-dependent parton physics relies on the QCD factorization theorem [25] and considerable advancements in global analyses [26-30] of experimental data. LQCD cannot calculate PDFs directly due to its Euclidean space formulation. QCD factorization can, however, connect $x$-dependent parton physics to a class of hadron matrix elements-"lattice cross sections" (LCSs) — that are 
calculable in LQCD and factorizable with perturbative matching. Several LQCD methods [31-37] have been proposed and developed to probe the light-cone structure of hadrons. These approaches have led to significant achievements in recent years, especially in determinations of flavor non-singlet distributions [38-47]. A proper quantification and mitigation of systematic errors and numerical artifacts present in these calculations and related theoretical challenges still require further insight and development (for a recent review, see [48]).

In this paper, we present an extraction of the $q_{\mathrm{v}}^{\pi}(x)$ from LCSs-LQCD-calculated pion matrix elements of two local, spacelike-separated and gauge-invariant currents [35,37]. These Lorentz covariant matrix elements of two currents spatially separated by a quark propagator are computable on a Euclidean lattice and have a well-defined continuum limit as the lattice spacing $a \rightarrow 0$. Calculations on four distinct lattice ensembles allows for estimation of systematic errors from finite lattice spacing, volume, and unphysical pion mass extrapolations. From parity and time-reversal invariance, this vector-axial (VA) current combination is antisymmetric and directly proportional to the $q_{\mathrm{v}}^{\pi}(x)$ with a perturbatively calculable coefficient function that matches this position space LCS to the $q_{\mathrm{v}}^{\pi}(x)$ in momentum space [43]. With both leading order (LO) and next-to-leading order (NLO) matching coefficients, we extract $q_{\mathrm{v}}^{\pi}(x)$ from the LQCD-calculated pion matrix elements and find that it is remarkably consistent with the same distribution extracted from experimental data over the entire range of $x$. We also find that our calculated NLO coefficient function, matching what is calculated in LQCD in position space to PDFs in momentum space, is very stable without the large logarithms that are often seen in the perturbatively calculated hard coefficients in momentum space.

The rest of this paper is organized as follows. In Sec. II, we first define the pion matrix elements that we calculate in LQCD, and introduce the factorization formalism to match the matrix elements in position space to the PDFs in momentum space. We then present our perturbative calculation, and provide results for the NLO matching coefficients. We demonstrate the effect of NLO matching coefficient and its perturbative stability in the factorized contribution to the pion matrix elements in position space in Sec. III. In Sec. IV, we explore the stability of the continuum limit of the LQCD-calculated pion matrix elements, and present the numerical extraction of $q_{\mathrm{v}}^{\pi}(x)$. We then present a discussion of our results in Sec. V, and finally, give our conclusions and outlook in Sec. VI.

\section{CALCULATION OF NEXT-TO-LEADING ORDER PERTURBATIVE KERNEL}

Following our previous work [43], we consider the following antisymmetrized matrix element in a hadron $h$ :

$$
\begin{aligned}
\sigma_{V A}^{[h], \mu \nu}(\xi, p)= & \xi^{4} Z_{V} Z_{A}\langle h(p)| T\left\{\left[\bar{\psi} \gamma^{\mu} \psi\right](\xi)\right. \\
& \left.\times\left[\bar{\psi} \gamma^{\nu} \gamma^{5} \psi\right](0)\right\}|h(p)\rangle+V \leftrightarrow A,
\end{aligned}
$$

where $\sigma_{V A}^{[h], \mu \nu}$ depends covariantly on the hadron momentum $p$ and spatial separation $\xi$ between the currents; $Z_{V, A}$ are the renormalization constants of the local currents determined in [49] for the ensembles used in this calculation. A Lorentz decomposition of Eq. (1) yields two scalar functions $T_{i=1,2}^{[h]}\left(\omega, \xi^{2}, p^{2}\right)$ where $\omega=-p \cdot \xi$ is the Ioffe time of the process [50], $T_{i}^{[h]}\left(\omega, \xi^{2}, p^{2}\right)=-T_{i}^{[h]}\left(-\omega, \xi^{2}, p^{2}\right)$ from parity and time-reversal invariance, and $T_{2}^{[h]}$ is power suppressed. For sufficiently small separations, $T_{1}^{[h]}$, which can be isolated by choosing $\mu=1$ and $\nu=2$, can be factorized [37]

$$
\begin{aligned}
T_{1}^{[h]}\left(\omega, \xi^{2}, p^{2}\right)= & \sum_{q} \int_{0}^{1} d x K\left(x \omega, \xi^{2}, x^{2} p^{2}, \mu^{2}\right) \\
& \times f_{q_{\mathrm{v}} / h}\left(x, \mu^{2}\right)+\mathcal{O}\left(\xi^{2} \Lambda_{\mathrm{QCD}}^{2}\right),
\end{aligned}
$$

where $f_{q_{\mathrm{v} / h}}\left(x, \mu^{2}\right) \equiv f_{q / h}\left(x, \mu^{2}\right)-f_{\bar{q} / h}\left(x, \mu^{2}\right)$ are valence PDFs, $\mu^{2}$ is the factorization scale, and the $K$ is perturbative matching coefficient with $K\left(x \omega, \xi^{2}, x^{2} p^{2}, \mu^{2}\right)=$ $-K\left(-x \omega, \xi^{2}, x^{2} p^{2}, \mu^{2}\right)$. Since $K$ depends on $\xi$, conventional techniques used to calculate matching coefficients in momentum space cannot be applied directly [51]. To perturbatively calculate $K\left(x \omega, \xi^{2}, 0, \mu^{2}\right)$ with an on-shell struck parton, $k^{2}=x^{2} p^{2}=0$, we could either calculate the matching coefficient directly in position space or introduce a "momentum space" matching coefficient with a " $D$-dimensional" Fourier transform

$$
\begin{aligned}
\tilde{T}_{1}^{[h]}\left(\tilde{\omega}, q^{2}\right) \equiv & \int \frac{d^{D} \xi}{\xi^{4}} e^{i q \cdot \xi} T_{1}^{[h]}\left(\omega, \xi^{2}, 0\right) \\
= & \int_{0}^{1} d x \tilde{K}\left(x \tilde{\omega}, q^{2}, \mu^{2}\right) f_{q_{\mathrm{v} / h}}\left(x, \mu^{2}\right) \\
& +\mathcal{O}\left(\Lambda_{\mathrm{QCD}}^{2} / q^{2}\right),
\end{aligned}
$$

where $D=4-2 \epsilon$ and $\tilde{\omega}=\frac{2 p \cdot q}{-q^{2}-i 0^{+}}$. With the perturbatively calculated $\tilde{K}$, we can obtain $K$ as

$K\left(x \omega, \xi^{2}, 0, \mu^{2}\right)=\xi^{4} \int \frac{d^{D} q}{(2 \pi)^{D}} e^{-i q \cdot \xi} \tilde{K}\left(x \tilde{\omega}, q^{2}, \mu^{2}\right)$.

To calculate $\tilde{K}$, we consider the matrix element of an on-shell quark state $q$ in Eq. (3), expand both sides in powers of the strong coupling $\alpha_{s}$, and keep up to NLO,

$\tilde{T}_{1}^{[q](0)}\left(\tilde{\omega}, q^{2}\right)=\int_{0}^{1} d x \tilde{K}^{(0)}\left(x \tilde{\omega}, q^{2}, \mu^{2}\right) f_{q_{\mathrm{v}} / q}^{(0)}\left(x, \mu^{2}\right)$, 


$$
\begin{aligned}
\tilde{T}_{1}^{[q](1)}\left(\tilde{\omega}, q^{2}\right)= & \int_{0}^{1} d x \tilde{K}^{(1)}\left(x \tilde{\omega}, q^{2}, \mu^{2}\right) f_{q_{\mathrm{v}} / q}^{(0)}\left(x, \mu^{2}\right) \\
& +\int_{0}^{1} d x \tilde{K}^{(0)}\left(x \tilde{\omega}, q^{2}, \mu^{2}\right) f_{q_{\mathrm{v}} / q}^{(1)}\left(x, \mu^{2}\right) .
\end{aligned}
$$

With the well-known $\overline{\mathrm{MS}}$ perturbative PDFs,

$$
\begin{aligned}
& f_{q_{\mathrm{v}} / q}^{(0)}\left(x, \mu^{2}\right)=\delta(1-x), \\
& f_{q_{\mathrm{v}} / q}^{(1)}\left(x, \mu^{2}\right)=-\frac{1}{\epsilon} \frac{(4 \pi)^{\epsilon}}{\Gamma(1-\epsilon)} \frac{\alpha_{s}}{2 \pi} C_{F}\left(\frac{1+x^{2}}{1-x}\right)_{+},
\end{aligned}
$$

$\tilde{K}^{(0)}$ and $\tilde{K}^{(1)}$ are determined by $\tilde{T}_{1}^{[q](0)}$ and $\tilde{T}_{1}^{[q](1)}$ using Eqs. (5) and (6). The $\tilde{T}_{1}^{[q](0)}$ and $\tilde{T}_{1}^{[q](1)}$ are obtained by calculating the two-current (VA) correlator up to $\mathcal{O}\left(\alpha_{s}\right)$ in $D$ dimension. Due to Ward-Takahashi identities for vector and axial-vector currents, UV divergences cancel out within one-loop diagrams and we do not need perturbative renormalization, which means $Z_{V}=Z_{A}=1$ in the perturbative calculation. One can also verify that perturbative collinear divergences from $\tilde{T}_{1}^{[q](1)}$ cancel exactly with $f_{q_{\mathrm{v}} / q}^{(1)}$ in Eq. (5), resulting in finite $\tilde{K}^{(0)}$ and $\tilde{K}^{(1)}$, and thus up to $\mathcal{O}\left(\alpha_{s}\right)$

$$
\begin{aligned}
& \tilde{K}\left(\tilde{\omega}, q^{2}, \mu^{2}\right) \\
&=\left\{\frac{1}{1+\tilde{\omega}}+\frac{\alpha_{s} C_{F}}{4 \pi}\left[\left(\frac{2+2 \tilde{\omega}^{2}}{\tilde{\omega}+\tilde{\omega}^{2}} \ln (1+\tilde{\omega})+\frac{3 \tilde{\omega}}{1-\tilde{\omega}^{2}}\right)\right.\right. \\
& \times \ln \left(\frac{\mu^{2}}{-q^{2}-i 0^{+}}\right)+\frac{5 \tilde{\omega}}{1-\tilde{\omega}^{2}}+\frac{2-2 \tilde{\omega}-\tilde{\omega}^{2}}{\tilde{\omega}+\tilde{\omega}^{2}} \ln (1+\tilde{\omega}) \\
&\left.\left.-\frac{1+\tilde{\omega}^{2}}{\tilde{\omega}+\tilde{\omega}^{2}} \ln ^{2}(1+\tilde{\omega})\right]\right\}-(\tilde{\omega} \rightarrow-\tilde{\omega}) .
\end{aligned}
$$

By performing a Fourier transform, we obtain

$$
\begin{aligned}
K\left(\omega, \xi^{2}, \mu^{2}\right)= & \frac{1}{\pi^{2} \omega}\left[K^{(0)}(\omega)+\frac{\alpha_{s} C_{\mathrm{F}}}{2 \pi}\left\{K^{(1,0)}(\omega)\right.\right. \\
& \left.\left.+K^{(1,1)}(\omega) \ln \left(-\xi^{2} \mu^{2} e^{2 \gamma_{E}} / 4\right)\right\}\right],
\end{aligned}
$$

with

$$
\begin{gathered}
K^{(0)}(\omega)=\omega \cos \omega, \\
K^{(1,0)}(\omega)=\omega \int_{0}^{1} d y \cos (y \omega)\left[\frac{1}{2} \delta(1-y)\right. \\
\left.-\left(\frac{2 \ln (1-y)}{1-y}-\frac{y^{2}-3 y+1}{1-y}\right)_{+}\right] \\
K^{(1,1)}(\omega)=-\omega \int_{0}^{1} d y \cos (y \omega)\left(\frac{1+y^{2}}{1-y}\right)_{+},
\end{gathered}
$$

where the leading order kernel $K^{(0)}(\omega)$ in Eq. (9) is the same as the result in [43]. After the integration over $y$, the NLO matching coefficient $K\left(\omega, \xi^{2}, \mu^{2}\right)$ is very stable and without large logarithms in $\omega$ in the region where the lattice QCD data points are available. Like the typical perturbatively calculated matching coefficients in momentum space, the NLO matching kernels $K^{(1,0)}(\omega)$ and $K^{(1,1)}(\omega)$, before the Fourier transform of $y$ into position space, have terms with the standard "+" prescription in Eq. (10). The existence of these + prescription terms is a natural result of perturbative cancelation of infrared (IR) divergences between the real and virtual contributions (or Feynman diagrams), and these terms have large logarithmic corrections at the point of the IR cancellation [52,53]. When these terms are directly convoluted with PDFs in momentum space to derive cross sections, a resummation of such large logarithmic perturbative corrections from the area of IR cancellation is needed to improve the perturbative stability of factorized cross sections so as to be better compared with experimental data near the kinematic threshold [11,54]. On the other hand, the QCD factorization proved for the LCSs [37] matches directly the hadron matrix elements calculated in position space to the PDFs in the momentum space, and is valid when the spatial separation $\xi$ of two currents is sufficiently small $\xi^{2} \ll$ $1 / \Lambda_{\mathrm{QCD}}^{2}$. It is this matching of matrix elements in position space to the PDFs in momentum space that helps reduce the perturbative sensitivity to the IR cancellation that takes place at a single point in phase space. As demonstrated in Ref. [37], the position space matching coefficient $K\left(\omega, \xi^{2}, \mu^{2}\right)$ is perturbatively analytic for all values of $\omega$ except $\omega \rightarrow \infty$. Technically, the Fourier transform over $y$ in Eq. (10) gives no $\log (\omega)$ terms to the $K\left(\omega, \xi^{2}, \mu^{2}\right)$ kernel when $\omega$ is in a perturbatively relevant region, and thus reduces the logarithmic perturbative sensitivity from the terms with the + prescription. With a small spatial separation between two currents required by the QCD factorization and the limited values of hadron momentum, the relevant $\omega$ is never too large in a practical lattice QCD calculation.

A convergence test of this NLO kernel is demonstrated in Sec. III. We highlight that a large hadron momentum $p$ alone does not automatically guarantee QCD factorization of the hadron matrix element in Eq. (1) into the PDFs - and the perturbative kernel, and contributions from the large $\xi$ region could invalidate the perturbative factorization $[37,43]$. It is the smallness of the spatial separation that defines the short-distance probe to see the particle nature of the partons inside a hadron and provides a required hard scale for the QCD factorization. Although not directly related to this calculation, the need to prove QCD factorization in momentum space is not new and is well known for the transverse momentum $k_{T}$ part of the transverse momentum dependent (TMD) factorization. The factorization formalism was proved in its conjugated position $b_{T}$ space, 
not in the momentum $k_{T}$ space [55], and the perturbative matching coefficients, as well as the evolution kernels, are calculated in position space and valid only for small $b_{T}$. The perturbative calculation method introduced in this paper can be used not only for the current-current operators, but also for operators defining quasi-PDFs [34] and reduced pseudo-ITDs [36] whose factorization to the PDFs are also valid for the region where the spatial separation between two active parton fields is small and much less than $1 / \Lambda_{\mathrm{QCD}}^{2}$. More importantly, our method is not restricted to NLO, but can be applied to any perturbative order [56]. The main subtlety of the method lies in the Fourier transformation, which must be done in $D$ dimensions as indicated in Eq. (4).

\section{EFFECT OF THE NEXT-TO-LEADING ORDER KERNEL ON THE IOFFE-TIME DISTRIBUTION}

To demonstrate the effect of the NLO kernel on the Ioffetime distribution (ITD), we select $\alpha_{s}=0.303$ at $\mu=2 \mathrm{GeV}$ and $-\xi^{2} \mu^{2}=1$ and compare in Fig. 1 the $K^{(0)}(\omega) / \omega$ and $K^{(1)}(\omega) / \omega$ effects for $\omega \neq 0$. The NLO corrections are tiny at small $\omega$ and increase very slowly towards large $\omega$; this can be partially understood from the ratio between $K^{(0)}$ and $K^{(1)}$ around $\omega=0$ :

$$
\frac{K^{(1)}}{K^{(0)}}=\frac{\alpha_{s}}{3 \pi}+\mathcal{O}\left(\omega^{2}\right) \approx 0.03+O\left(\omega^{2}\right)
$$

It is important to note that as an asymptotic series, the relative size of $K^{(1)} / K^{(0)}$ as a function of $\omega$ actually diverges as $\omega \rightarrow \infty$, but, only a small range of $\omega$ is relevant for the convolution with PDFs in Eq. (1). What is important is the size of their convolutions with the PDFs in the relevant Ioffe-time window of the lattice QCD data while keeping $\xi$ small. Therefore, it is also useful to demonstrate

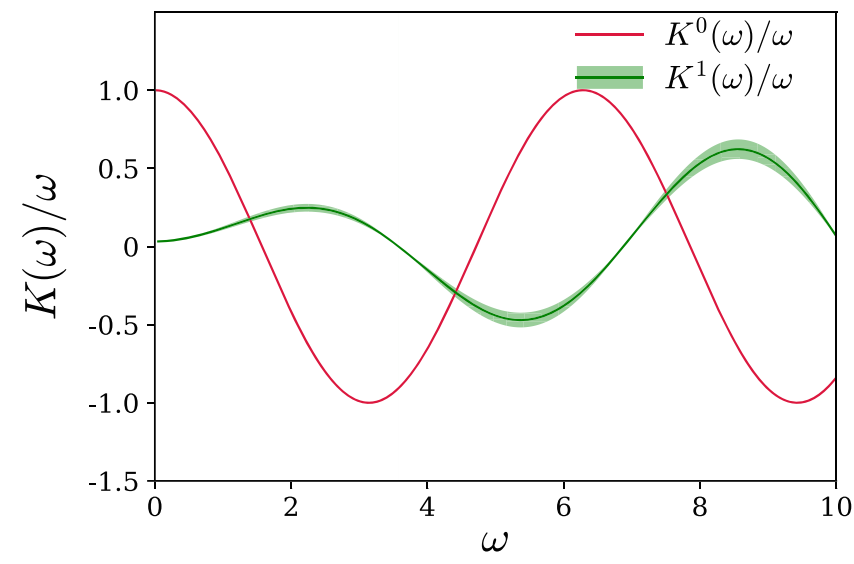

FIG. 1. A comparison between $K^{(0)}(\omega) / \omega$ and $K^{(1)}(\omega) / \omega$ for $\alpha_{s}(\mu=2 \mathrm{GeV})=0.303$ and $-\xi^{2} \mu^{2}=1$. The uncertainty in $K^{(1)}(\omega) / \omega$ is obtained by a $10 \%$ variation in $\alpha_{s}$.

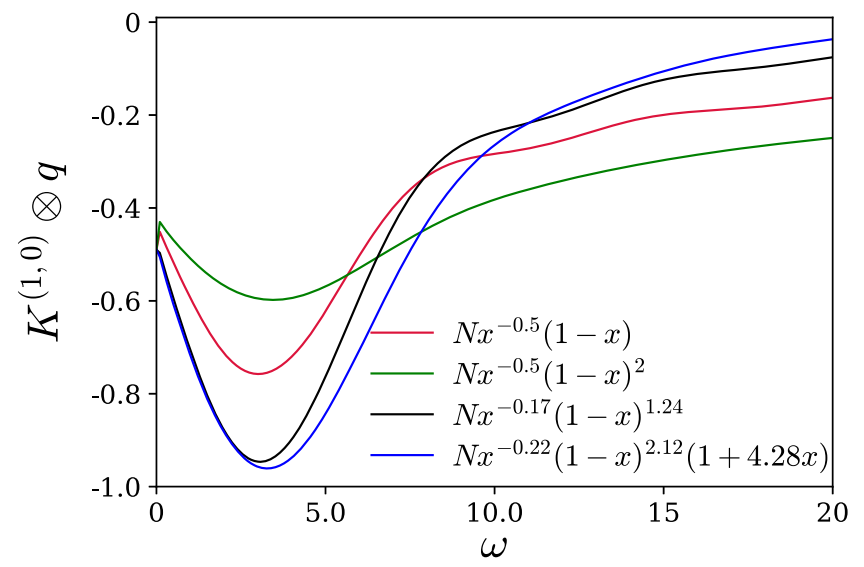

FIG. 2. The convolution of the $K^{(1,0)}$ kernel with model PDFs.

the effect the NLO kernels could have with various model PDFs in the Ioffe-time space. The convolutions

$$
K^{(1, i)} \otimes q(\omega)=\int d x \frac{1}{x \omega} K^{(1, i)}(x \omega) q(x)
$$

with $i=0,1$ for a few PDFs are shown in Figs. 2 and 3, respectively. Each of the convolutions have similar features. These convolutions represent the difference between the LCS and the ITD, applying the appropriate factors proportional to $\alpha_{s}$ and $\ln \left(-\xi^{2} \mu^{2} e^{2 \gamma_{E}} / 4\right)$. The convolutions all rise to a peak around $\omega \sim 4.0$ and begin to decay to 0 . The NLO effects are most significant at the highest Ioffe-time range available to our calculations but the corrections will be smaller for large Ioffe times. These convolutions demonstrate a reassuring feature of the position space matching. These convolutions are at the largest $O(1)$ which means the NLO term will be $O\left(\alpha_{s}\right)$ for the entire region of Ioffe time. These convolutions can be compared with those for matching the reduced pseudo-ITD to the PDF in [47].

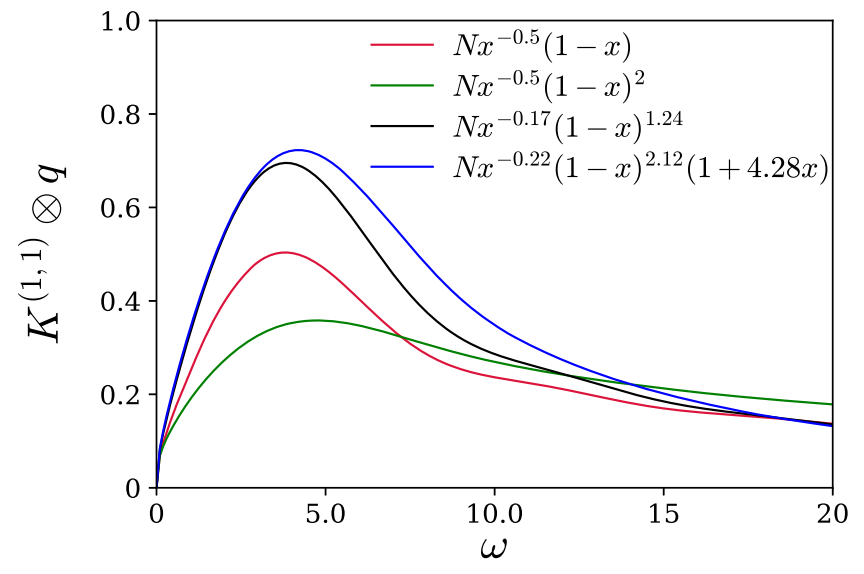

FIG. 3. The convolution of the $K^{(1,1)}$ kernel with model PDFs. 


\section{NUMERICAL RESULTS \& EXTRACTION OF THE $q_{\mathrm{v}}^{\pi}(x)$}

The LQCD calculation of the pion matrix element in Eq. (1) is carried out on four different $2+1$ flavor QCD ensembles (listed in Table I) using the isotropic-clover fermion action generated by the JLab/W\&M Collaboration [57]. We refer to [43] for details about the implementation of a modified sequential source technique, and a combination of Jacobi and momentum smearing to obtain matrix elements for a given momentum $p$ and spatial separation $\xi$ between the currents. In this calculation of the forward matrix elements, the pion source-sink separation $T$ is systematically increased, while holding fixed the current insertion time $t=T / 2$, ensuring identical excited-state contamination from both source and sink sides. To extract the desired matrix elements, we assume the following forms of two- and four-point correlation functions:

$$
\begin{aligned}
& C_{2 p t}(T)=A e^{-m_{0} T} \\
& C_{4 p t}(T)=e^{-m_{0} T}\left(B+D e^{-\Delta m T}\right),
\end{aligned}
$$

and perform simultaneous correlated fits to the two- and four-point functions. We verify that the value of the groundstate energy $m_{0}$ obtained from this simultaneous fit is consistent with that obtained from $C_{2 p t}(T)$ alone and also agrees with the energy-momentum dispersion relation.

In Fig. 4, we present fit results of the ratio $C_{4 p t}(T) / e^{-m_{0} T}$ on the ensembles $a 94 m 278$ and $a 94 m 358$ for momenta in the range $p \in\{0.41-1.65\} \mathrm{GeV}$ and current separation $\xi=3 a$, both $p$ and $\xi$ in along the $z$ direction, to demonstrate how reliably we can extract the asymptotic value of $B$, and hence the ITD from $B / A$. The numerical challenges manifest in this formalism are reflected in the signal-tonoise ratio $(S / N)$ of the largest momentum $p=1.65 \mathrm{GeV}$ relative to that of the smallest $p=0.41 \mathrm{GeV}$; the former is nearly 3 times smaller. Despite this, we can fit these data up to at least $T=14(\sim 1.32 \mathrm{fm})$ even for the largest momentum $p=1.65 \mathrm{GeV}$ on the lightest pion mass $m_{\pi}=$ $278 \mathrm{MeV}$ ensemble. In all the fits, we use the time window such that $S / N \geq 1$. The Wilson clover fermion action explicitly connects adjacent lattice sites, introducing spurious contact terms in the $\xi=a$ matrix element signals. These data are consequently neglected from our analysis.

TABLE I. Parameters for each gauge ensemble used in this work: lattice spacing, pion mass, spatial and temporal sizes, and number of configurations used.

\begin{tabular}{lcccc}
\hline \hline ID & $a(\mathrm{fm})$ & $m_{\pi}(\mathrm{MeV})$ & $L^{3} \times N_{t}$ & $N_{\text {cfg }}$ \\
\hline$a 127 m 413$ & $0.127(2)$ & $413(4)$ & $24^{3} \times 64$ & 2124 \\
$a 127 m 413 L$ & $0.127(2)$ & $413(5)$ & $32^{3} \times 96$ & 490 \\
$a 94 m 358$ & $0.094(1)$ & $358(3)$ & $32^{3} \times 64$ & 417 \\
$a 94 m 278$ & $0.094(1)$ & $278(4)$ & $32^{3} \times 64$ & 503 \\
\hline \hline
\end{tabular}
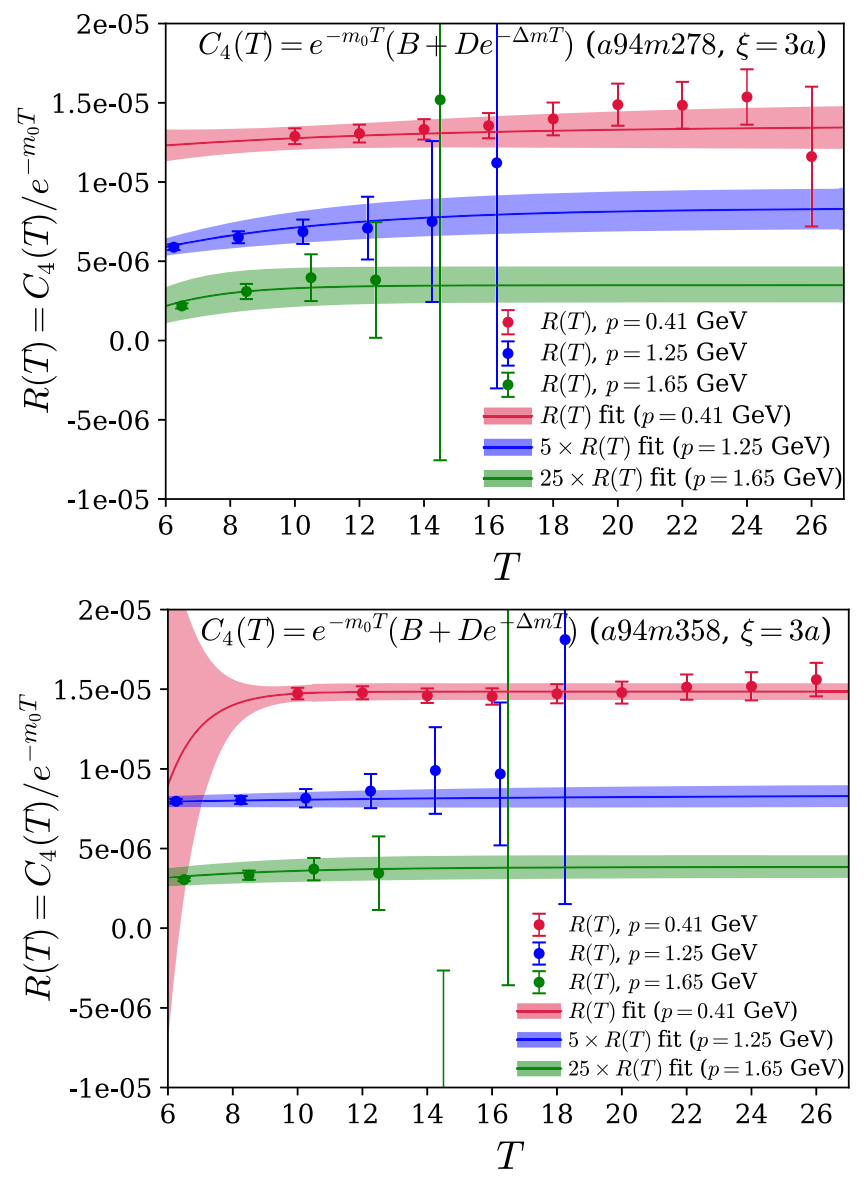

FIG. 4. Removal of the leading ground-state time dependence exposes the desired matrix elements in the large $T$ limit, shown here for ensembles $a 94 m 278$ (above) and $a 94 m 358$ (below) for current separations $\xi=3 a$. High momenta data rescaled for $S / N$ comparison.

The matrix elements computed across the four gauge ensembles are shown in Fig. 5. We only include $|\xi| \leq$ $0.56 \mathrm{fm}$ in our analysis so that $\xi$ is sufficiently smaller than $\Lambda_{\mathrm{QCD}}^{-1}$, thereby ensuring the validity of the short-distance factorization and minimizing higher-twist contributions from large $\xi$. Exploiting the analyticity of the LCS $T_{1}^{\pi}\left(\omega, \xi^{2}\right)=\sigma_{V A}^{12}\left(\omega, \xi^{2}\right)$ in $\omega$ and denoting $\sigma_{V A}^{12}\left(\omega, \xi^{2}\right) \equiv$ $\sigma_{V A}\left(\omega, \xi^{2}\right)$ in the rest of the article and figures, we obtain the functionally unknown ITD using a flexible $z$-expansion fit [58,59] supplemented with chiral, continuum, finite volume [60] and higher-twist corrections:

$$
\begin{aligned}
\sigma_{V A}\left(\omega, \xi^{2}\right)= & \sum_{k=0}^{k_{\max }=4} \lambda_{k} \tau^{k}+b_{1}\left(m_{\pi}-m_{\pi, \text { physical }}\right)+b_{2} a \\
& +b_{3} \xi^{2}+b_{4} a^{2} p^{2}+b_{5} e^{-m_{\pi}(L-\xi)} \\
& \text { where } \tau=\frac{\sqrt{\omega_{\text {cut }}+\omega}-\sqrt{\omega_{\text {cut }}}}{\sqrt{\omega_{\text {cut }}+\omega}+\sqrt{\omega_{\text {cut }}}}
\end{aligned}
$$




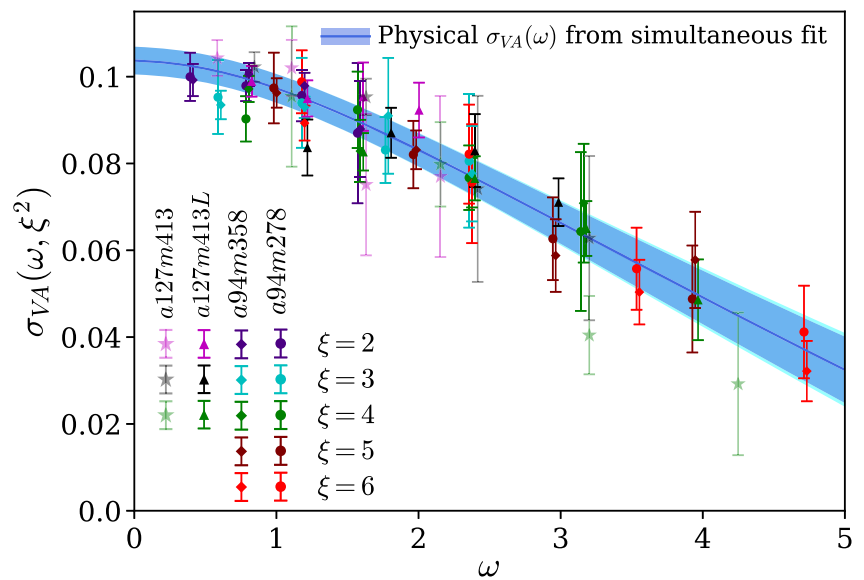

FIG. 5. Simultaneous fit to the antisymmetric VA current matrix elements on four different ensembles. The blue band indicates the ITD in the physical limit. The outer cyan band shows the combined statistical and systematic uncertainties of fit (14) added in quadrature.

and $m_{\pi \text {,physical }} \simeq 0.14 \mathrm{GeV}$ is the physical pion mass. Higher-order terms $\left(k_{\max }>4\right)$ have no statistical significance and are not considered.

The correction terms of Eq. (14) are selected based on each being the dominant contribution of its type. We consider now different possible correction terms in the fit to Eq. (14), such as $a^{2}, m_{\pi}^{2}, L e^{-m_{\pi}(L-\xi)}, \sqrt{L} e^{-m_{\pi}(L-\xi)}$. These corrections are presented in Table II. The second column indicates the value of the fitted coefficient of the correction terms and the $\lambda_{k}$ columns indicate the effect of these corrections on the $z$-expansion fit parameters used to obtain the physical limit $\sigma_{V A}(\omega)$ distribution. We note that for all additionally considered corrections, the effect is indeed observed to be less than the original corrections of Eq. (14) and the determination of $q_{\mathrm{v}}^{\pi}(x)$ remains unaffected.

In addition, as seen in Fig. 5 the effects of other possible correction terms such as $m_{\pi}^{2}, a^{2}, L e^{-m_{\pi}(L-\xi)}, \sqrt{L} e^{-m_{\pi}(L-\xi)}$ are observed to be very mild. We choose $\omega_{\text {cut }}=1.0$ as used in [61]; other choices of $\omega_{\text {cut }}$ were observed to have no effect on the final band in the physical limit and vanishing higher-twist $\mathcal{O}\left(\xi^{2}\right)$ contributions. The blue band in Fig. 5 shows such $\sigma_{V A}(\omega)$ distribution after $b_{i}$ corrections in Eq. (14) are subtracted, and where the error band is determined from the $\lambda_{k}$ covariances. The fit yields $\lambda_{0}=0.104(3), \quad \lambda_{1}=-0.006(3), \quad \lambda_{2}=-0.029(9)$,

$\lambda_{3}=-0.907(404), \quad \lambda_{4}=0.124(136)$,

$b_{1}=0.174(96), \quad b_{2}=-0.083(43), \quad b_{3}=-0.0004(7)$,

$b_{4}=0.007(8), \quad b_{5}=0.102(51)$

with $\chi^{2} /$ d.o.f $=1.20$. As can be seen in Fig. 5, there appears to be completely negligible $\xi$ effects either higher twist or Dokshitzer-Gribov-Lipatov-Altarelli-Parisi, DGLAP [62-64]. Therefore, we will assign the $\xi=2 \times 0.094 \mathrm{fm}$, the shortest $\xi$ used in this study in the factorization formula (8) while matching the position space LCS to $q_{\mathrm{v}}^{\pi}(x)$ distribution. With the physical $\sigma_{V A}(\omega)$ distribution in hand, we can immediately extract the physical $q_{\mathrm{v}}^{\pi}(x)$ with no further extrapolations.

The extraction of $q_{\mathrm{v}}^{\pi}(x)$ is achieved by numerically evaluating the convolution of the NLO kernel equation (8) and the following phenomenologically motivated functional forms of the PDF:

$$
q_{\mathrm{v}}^{\pi}(x)=\frac{x^{\alpha}(1-x)^{\beta}(1+\gamma x)}{B(\alpha+1, \beta+1)+\gamma B(\alpha+2, \beta+1)}
$$

using the library ROOT [65]. The high correlation of the sampled $\sigma_{V A}(\omega)$ data guarantees that increasing the sampling density or varying the number of derived pseudodata samples will have no impact on the $q_{\mathrm{v}}^{\pi}(x)$ fit parameters.

The parameters in the PDF parametrization equation (17) are determined by fitting the convolution of the model PDF and the NLO perturbative kernel to $\sigma_{V A}(\omega)$ in a manner similar to Ref. [61], where the Ioffe-time zero point is fixed in this calculation by the LO + NLO perturbative kernel. The isolation of $\sigma_{V A}(\omega)$ is a multistep process, and begins by performing a correlated fit of lattice data from all four ensembles according to Eq. (14). This yields $\sigma_{V A}\left(\omega, \xi^{2}\right)$ plus corrections. Removing the $b_{i}$ corrections from the obtained $\sigma_{V A}\left(\omega, \xi^{2}\right)$ distribution, we obtain the blue band indicated by $\sigma_{V A}(\omega)$-now in the physical limit. The covariance matrix of the $\lambda_{k}$ coefficients from the correlated $z$-expansion fit provides an error estimate of the $\sigma_{V A}(\omega)$ physical distribution. We choose 30 correlated data points from the continuum band of $\sigma_{V A}(\omega)$, equally spaced in the Ioffe-time interval $\omega \in[0-4.71]$; a number in accordance with the 20 data points available from the $a 94 m 278$ and $a 94 m 358$ lattice ensembles. Using the mean and

TABLE II. Fit parameters of different correction terms in fit Eq. (14) for the investigation of systematic uncertainties in $\sigma_{V A}(\omega)$.

\begin{tabular}{lccccccc}
\hline \hline Correction term & Fit coefficient & $\lambda_{0}$ & $\lambda_{1}$ & $\lambda_{2}$ & $\lambda_{3}$ & $\lambda_{4}$ & $\chi^{2} /$ d.o.f. \\
\hline$a^{2}$ & $-0.049(34)$ & $0.0104(3)$ & $-0.006(3)$ & $-0.028(9)$ & $-0.901(391)$ & $0.124(135)$ & 1.26 \\
$\left(m_{\pi}^{2}-m_{\pi, \text { physical }}^{2}\right)$ & $0.15(12)$ & $0.0104(3)$ & $-0.006(3)$ & $-0.029(10)$ & $-0.926(388)$ & $0.118(132)$ & 1.18 \\
$L e^{-m_{\pi}(L-\xi)}$ & $0.007(3)$ & $0.0104(3)$ & $-0.006(3)$ & $-0.028(10)$ & $-0.915(402)$ & $0.121(136)$ & 1.22 \\
$\sqrt{L} e^{-m_{\pi}(L-\xi)}$ & $0.026(14)$ & $0.0104(3)$ & $-0.006(3)$ & $-0.029(10)$ & $-0.914(403)$ & $0.121(136)$ & 1.21 \\
\hline \hline
\end{tabular}


covariance matrix of these data points, we create 200 Gaussian distributed pseudodata samples with appropriate correlations and perform the following numerical fit:

$$
\sigma_{V A}(\omega)=\int_{0}^{1} d x K^{\mathrm{LO}+\mathrm{NLO}}(x, \omega) q_{\mathrm{v}}^{\pi}(x)
$$

to obtain $q_{\mathrm{v}}^{\pi}(x)$. As these discrete values resulting from the fit in Eq. (14) are highly correlated, the addition of more discrete data points from the fitted $\sigma_{V A}(\omega)$ distribution does not improve the outcome of the $q_{\mathrm{v}}^{\pi}(x)$ fit parameters. We confirmed this by increasing the number of $\sigma_{V A}(\omega)$ sampling points to 100. A similar result is obtained if one chooses 20 sampling points or less, as was done in our previous work (Ref. [43]). One can also see that increasing or decreasing the number of pseudodata samples from 200 will not have any impact on the $q_{\mathrm{v}}^{\pi}(x)$ fit parameters, again due to the $\sigma_{V A}(\omega)$ data correlations. What is required to improve the $q_{\mathrm{V}}^{\pi}(x)$ fit parameters is a larger range of Ioffe time. In the above fit, we have used the constraints $\alpha \leq 0$ and $\beta \leq 4$.

For the above fit, we use $\alpha_{s}=0.303$ at the initial scale $\mu_{0}=2 \mathrm{GeV}$ [66]. Systematic uncertainties in each PDF parameter set are estimated by a $10 \%$ variation in $\alpha_{s}$ as in [61]. The 2-parameter fit, by fixing $\gamma=0$ in Eq. (17), yields

$\alpha=-0.17(7)_{\text {stat }}(2)_{\text {sys }}, \quad \beta=1.24(22)_{\text {stat }}(7)_{\text {sys }}$

with $\chi^{2} /$ d.o.f $=1.41$. Stated uncertainties are statistical (systematic) first (second). In a 3-parameter fit, with an unconstrained $\gamma$, we obtain

$$
\begin{aligned}
& \alpha=-0.22(11)_{\text {stat }}(3)_{\text {sys }}, \quad \beta=2.12(56)_{\text {stat }}(14)_{\text {sys }}, \\
& \gamma=4.28(1.73)_{\text {stat }}(25)_{\text {sys }}
\end{aligned}
$$

with $\chi^{2} /$ d.o.f $\approx 1.29$. The present calculation has achieved a better statistical precision in the $\beta$ value compared to the

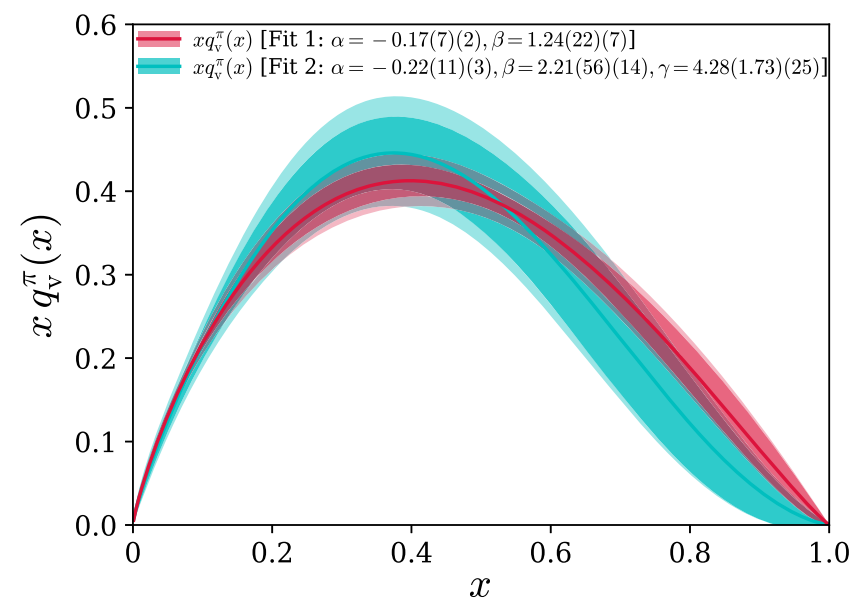

FIG. 6. The pion valence quark distribution obtained from fitting the convolution of $q_{\mathrm{v}}^{\pi}(x)$ and the NLO perturbative kernel (8) to the determined $\sigma_{V A}(\omega)$ distribution in the fit Eq. (14). Fits 1 and 2 label the 2- and 3-parameter functional forms in Eq. (17). previous LCS determination [43] where it was found in a 3 -parameter fit $\beta=1.93(68)$. Inclusion of an additional $\rho \sqrt{x}$-term in (17) was found to be consistent with zero. Commensurate $\chi^{2} /$ d.o.f between fits (19) and (20) limits the selection of one fit over another based solely on the goodness of the fit. These fits are shown in Fig. 6. We elected not to extrapolate our ITD obtained from our $z^{-}$ expansion fit beyond the largest Ioffe time $\omega=4.71$ when determining the PDF. It has been shown [67] when using sophisticated inversion methods that the large- $x$ behavior is well reproduced even with the limited range in Ioffe time.

\section{DISCUSSION}

As shown in Fig. 7, extrapolating the central value of the $\sigma_{V A}(\omega)$ distribution from the $z$-expansion fit (blue) and the associated 2- (red) and 3-parameter (cyan) fits reveals that precise LQCD data at large $\omega$ are required to distinguish between different large- $x$ behaviors of $q_{\mathrm{v}}^{\pi}(x)$. We validate our PDF fitting procedure by reconstructing the $\sigma_{V A}(\omega)$ distribution by convolving the NLO kernel with the PDFs obtained from the pseudodata samples. The $\sigma_{V A}(\omega)$ distribution reconstructed from the 2-parameter fit underestimates the uncertainty of the distribution in the physical limit by about $8 \%-12 \%$ for $\omega>4$, and starts to deviate from the blue band as $\omega$ increases. For a fixed $\alpha$, one can show that the ITD falls off faster for a smaller $\beta$ as a function of $\omega$ compared to that for a larger $\beta$ in a 2 or 3 or more parameter PDF functional form. Therefore, precise data at higher Ioffe time $(\omega \sim 8-10)$ will provide a better discrimination between different $\beta$ values in a future LCS calculation.

While PDFs can minimally be described by the $x^{\alpha}(1-x)^{\beta}$ functional form, encompassing the Regge theory [68] and pQCD based power counting rules [69], modern global analyses [26-28] inform our decision to

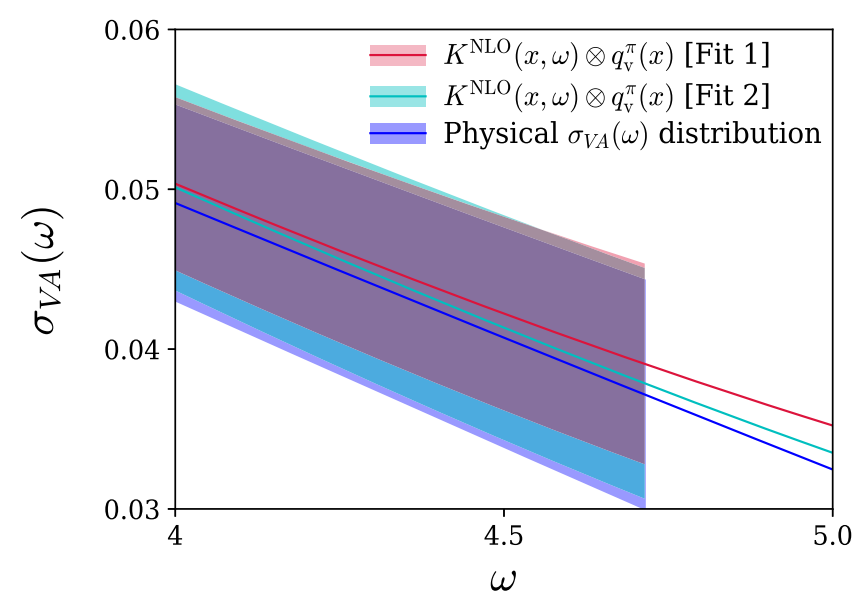

FIG. 7. A comparison of the reconstructed $\sigma_{V A}(\omega)$ distribution using Eq. (18) for $4.0<\omega<5.0$ from the PDF fits and that obtained from (14). Fits 1 and 2 label the 2- and 3-parameter functional forms in Eq. (17). 


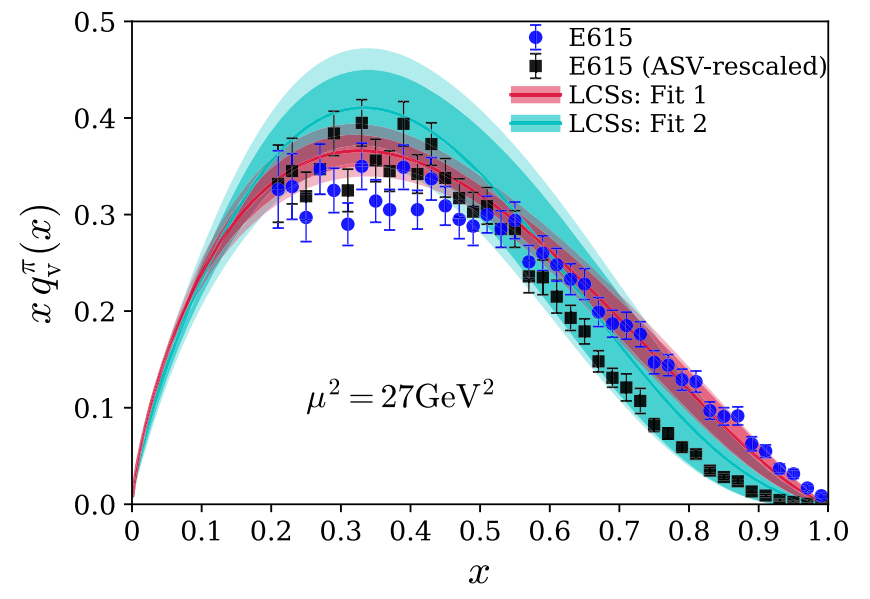

FIG. 8. Comparison of pion $x q_{\mathrm{v}}^{\pi}(x)$ distribution obtained from this calculation with the $x q_{\mathrm{v}}^{\pi}(x)$ distributions extracted from the experimental Drell-Yan cross sections. The blue data points are from LO analysis [3] and the "ASV-rescaled" black data points compiled from [71] are the E615 rescaled data according to analysis [11].

allow for an interpolating function between these small- $x$ and large- $x$ regions and thus a better and less biased description of PDFs. In particular, the fit (20) includes the possibility of $\gamma=0$ and is more flexible.

For a comparison with global fits of $q_{\mathrm{v}}^{\pi}(x)$, we evolve our extracted PDF sets to a scale of $\mu^{2}=27 \mathrm{GeV}^{2}$, from an initial scale $\mu_{0}=2 \mathrm{GeV}$ shown in Fig. 6, large enough for the validity of factorization. Figure 8 shows a comparison with the PDF extraction using LO factorization of the E615 data [3], which shows a $(1-x)$ large- $x$ behavior, and the analysis [11] where the next-to-leading-logarithmic threshold soft-gluon resummation effects $[52,53]$ are included in the calculation of the Drell-Yan cross section, which shows a softer $(1-x)^{2}$ falloff. A comparison between the pion PDFs obtained from previous lattice calculations using the LCS [43], quasi-PDFs [45,70], and pseudo-PDFs [61] methods can be found in [61].

We need QCD factorization and perturbatively calculated matching coefficients to enable us to extract the PDFs since they are neither direct physical observables, nor directly calculable in lattice QCD. However, QCD factorization is an approximation, and power corrections to the factorization formalism are likely more important when the observable, such as the Drell-Yan cross section, or the LQCD-calculated hadron matrix element, is pushed to the edge of phase space where $x \rightarrow 1$. On the other hand, we can get some information on the $x \rightarrow 1$ behavior of PDFs from the convolution of the factorized formalism, by measuring the physical cross sections or calculating the hadron matrix elements in LQCD not too close to the edge of phase space. However, the garnered information on $x \sim 1$ will be mild since the contribution from this region is much smaller than that from the smaller $x$ regions. This is exactly the reason why PDFs extracted from world data in QCD global analyses have a large uncertainty as $x \rightarrow 1$. Although it might be difficult to pin down the exact "power of $(1-x)$ " of the pion PDFs, the extraction of PDFs from future improved LQCD calculations of good hadron matrix elements, the LCSs that are calculable in LQCD and factorizable to PDFs, might help improve the accuracy of determining this "power," since the matching coefficients for LCSs in position space are more perturbatively stable at larger $x$ than the momentum-space matching coefficients for experimentally measured cross sections.

\section{CONCLUSION AND OUTLOOK}

In this paper we have presented the first LCS calculation of $q_{\mathrm{v}}^{\pi}(x)$ that incorporates results on four gauge ensembles, among these the lightest pion mass used in any lattice QCD calculation to access $q_{\mathrm{v}}^{\pi}(x)$, as well as the first derivation of NLO matching coefficients from position space directly to momentum space. The $q_{\mathrm{v}}^{\pi}(x)$ extracted from our LQCD calculation is remarkably consistent with that extracted from experimental data. Given that the NLO matching coefficient $K$ is very stable and without large threshold logarithms that are often seen in momentum space matching coefficients, our approach, plus future gauge ensembles with smaller lattice spacings, has the unique potential to provide a better determination of the "power of $(1-x)$ " of the $q_{\mathrm{v}}^{\pi}(x)$ distribution as $x \rightarrow 1$.

vCentral to this endeavor are calculations in the near future with finer lattice spacings. With a simpler nonperturbative UV renormalization, different choices of current combinations, and the nontrivial hadron-independent and stable NLO matching coefficients, the LCS formalism with two-current correlators is well equipped to unravel the enigmatic structure of the pion and other hadrons, especially those that are difficult, if not impossible, to study experimentally, complementary to other approaches, such as the quasi- and pseudo-PDFs approaches.

\section{ACKNOWLEDGMENTS}

We thank Carl Carlson, Martha Constantinou, Luka Leskovec, Tianbo Liu, Wayne Morris, Anatoly Radyushkin, Nobuo Sato, and Jian-Hui Zhang who provided insights and expertise that greatly assisted this research. This work is supported by Jefferson Science Associates, LLC under U.S. DOE Contract No. DEAC05-06OR23177 within the framework of the TMD Collaboration. We acknowledge the facilities of the USQCD Collaboration used for this research in part, which are funded by the Office of Science of the U.S. Department of Energy. This material is based in part upon work supported by a grant from the Southeastern Universities Research Association (SURA) under an appropriation from the Commonwealth of Virginia. This work used the Extreme Science and Engineering Discovery Environment (XSEDE), which is supported by National Science Foundation Grant No. ACI-1548562 [72], and the Texas 
Advanced Computing Center (TACC) at the University of Texas at Austin for providing HPC resources on Frontera that have contributed to the results in this paper. This work was performed in part using computing facilities at William and Mary which were provided by contributions from the National Science Foundation (MRI Grant No. PHY1626177), the Commonwealth of Virginia Equipment Trust Fund and the Office of Naval Research. In addition, this work used resources at National Energy Research Scientific Computing Center (NERSC), a DOE Office of Science User Facility supported by the Office of Science of the U.S. Department of Energy under Contract No. DEAC02-05CH11231, as well as resources of the Oak Ridge Leadership Computing Facility at the Oak Ridge National Laboratory, which is supported by the Office of Science of the U.S. Department of Energy under Contract No. DEAC05-00OR22725 (ALCC Project No. NPH134). The configurations used in this work were generated under INCITE. The software codes CHROMA [73], QUDA [74,75] and QPhiX [76] were used. The authors acknowledge support from the U.S. Department of Energy, Office of Science, Office of Advanced Scientific Computing Research and Office of Nuclear Physics, Scientific Discovery through Advanced Computing (SciDAC) program. Also acknowledged is support from the U.S. Department of Energy Exascale Computing Project. C.E. is supported in part by the U.S. Department of Energy under Contract No. DE-FG02-04ER41302 and a Department of Energy Office of Science Graduate Student Research fellowship, through the U.S. Department of Energy, Office of Science, Office of Workforce Development for Teachers and Scientists, Office of Science Graduate Student Research (SCGSR) program. Y. M. is supported in part by the National Natural Science Foundation of China under Grants No. 11875071 and No. 11975029. K. O. acknowledges support in part by the U.S. Department of Energy through Grant No. DEFG02-04ER41302, by STFC consolidated Grant No. ST/ P000681/1.
[1] J. Badier et al. (NA3 Collaboration), Experimental determination of the pi meson structure functions by the Drell-Yan mechanism, Z. Phys. C 18, 281 (1983).

[2] B. Betev et al. (NA10 Collaboration), Differential crosssection of high mass muon pairs produced by a $194-\mathrm{GeV} / c \pi^{-}$ beam on a tungsten target, Z. Phys. C 28, 9 (1985).

[3] J.S. Conway et al., Experimental study of muon pairs produced by $252-\mathrm{GeV}$ pions on tungsten, Phys. Rev. D 39, 92 (1989).

[4] S. Chekanov et al. (ZEUS Collaboration), Leading neutron production in e + p collisions at HERA, Nucl. Phys. B637, 3 (2002).

[5] F. D. Aaron et al. (H1 Collaboration), Measurement of leading neutron production in deep-inelastic scattering at HERA, Eur. Phys. J. C 68, 381 (2010).

[6] J. F. Owens, $Q^{2}$ dependent parametrizations of pion parton distribution functions, Phys. Rev. D 30, 943 (1984).

[7] P. Aurenche, R. Baier, M. Fontannaz, M. N. KienzleFocacci, and M. Werlen, The gluon content of the pion from high $p_{T}$ direct photon production, Phys. Lett. B 233, 517 (1989).

[8] P. J. Sutton, A. D. Martin, R. G. Roberts, and W. J. Stirling, Parton distributions for the pion extracted from Drell-Yan and prompt photon experiments, Phys. Rev. D 45, 2349 (1992).

[9] M. Gluck, E. Reya, and A. Vogt, Pionic parton distributions, Z. Phys. C 53, 651 (1992).

[10] K. Wijesooriya, P. E. Reimer, and R. J. Holt, The pion parton distribution function in the valence region, Phys. Rev. C 72, 065203 (2005).

[11] M. Aicher, A. Schafer, and W. Vogelsang, Soft-Gluon Resummation and the Valence Parton Distribution
Function of the Pion, Phys. Rev. Lett. 105, 252003 (2010).

[12] P. C. Barry, N. Sato, W. Melnitchouk, and C. R. Ji, First Monte Carlo Global QCD Analysis of Pion Parton Distributions, Phys. Rev. Lett. 121, 152001 (2018).

[13] G. R. Farrar and D. R. Jackson, The Pion Form-Factor, Phys. Rev. Lett. 43, 246 (1979).

[14] E. L. Berger and S. J. Brodsky, Quark Structure Functions of Mesons and the Drell-Yan Process, Phys. Rev. Lett. 42, 940 (1979).

[15] T. Shigetani, K. Suzuki, and H. Toki, Pion structure function in the Nambu and Jona-Lasinio model, Phys. Lett. B 308, 383 (1993).

[16] R. M. Davidson and E. R. Arriola, Structure functions of pseudoscalar mesons in the SU(3) NJL model, Phys. Lett. B 348, 163 (1995).

[17] M. B. Hecht, C. D. Roberts, and S. M. Schmidt, Valence quark distributions in the pion, Phys. Rev. C 63, 025213 (2001).

[18] C. Chen, L. Chang, C. D. Roberts, S. Wan, and H. S. Zong, Valence-quark distribution functions in the kaon and pion, Phys. Rev. D 93, 074021 (2016).

[19] G. F. de Téramond, T. Liu, R. S. Sufian, H. G. Dosch, S. J. Brodsky, A. Deur (HLFHS Collaboration), Universality of Generalized Parton Distributions in Light-Front Holographic QCD, Phys. Rev. Lett. 120, 182001 (2018).

[20] K. D. Bednar, I. C. Cloét, and P. C. Tandy, Distinguishing Quarks and Gluons in Pion and Kaon PDFs, Phys. Rev. Lett. 124, 042002 (2020).

[21] M. Ding, K. Raya, D. Binosi, L. Chang, C. D. Roberts, and S. M. Schmidt, Symmetry, symmetry breaking, and pion parton distributions, Phys. Rev. D 101, 054014 (2020). 
[22] D. Adikaram et al. (Hall A and SBS Collaboration Proposal), Measurement of tagged deep inelastic scattering (TDIS), Report No. PR12-15-006, 2015.

[23] B. Adams et al., Letter of intent: A new QCD facility at the M2 beam line of the CERN SPS (COMPASS++/AMBER), arXiv: 1808.00848.

[24] A. C. Aguilar et al., Pion and kaon structure at the electronion collider, Eur. Phys. J. A 55, 190 (2019).

[25] J. C. Collins, D. E. Soper, and G. F. Sterman, Factorization of hard processes in QCD, Adv. Ser. Dir. High Energy Phys. 5, 1 (1989).

[26] L. A. Harland-Lang, A. D. Martin, P. Motylinski, and R. S. Thorne, Parton distributions in the LHC era: MMHT 2014 PDFs, Eur. Phys. J. C 75, 204 (2015).

[27] S. Dulat, T.-J. Hou, J. Gao, M. Guzzi, J. Huston, P. Nadolsky, J. Pumplin, C. Schmidt, D. Stump, and C.-P. Yuan, New parton distribution functions from a global analysis of quantum chromodynamics, Phys. Rev. D 93, 033006 (2016).

[28] R. D. Ball et al. (NNPDF Collaboration), Parton distributions from high-precision collider data, Eur. Phys. J. C 77, 663 (2017).

[29] S. Alekhin, J. Blümlein, S. Moch, and R. Plačakytè, Parton distribution functions, $\alpha_{s}$, and heavy-quark masses for LHC Run II, Phys. Rev. D 96, 014011 (2017).

[30] J. J. Ethier, N. Sato, and W. Melnitchouk, First Simultaneous Extraction of Spin-Dependent Parton Distributions and Fragmentation Functions from a Global QCD Analysis, Phys. Rev. Lett. 119, 132001 (2017).

[31] K. F. Liu and S. J. Dong, Origin of Difference between $\bar{d}$ and $\bar{u}$ Partons in the Nucleon, Phys. Rev. Lett. 72, 1790 (1994).

[32] V. Braun and D. Mueller, Exclusive processes in position space and the pion distribution amplitude, Eur. Phys. J. C 55, 349 (2008).

[33] R. Horsley, R. Millo, Y. Nakamura, H. Perlt, D. Pleiter, P. E. L. Rakow, G. Schierholz, A. Schiller, F. Winter, and J. M. Zanotti (QCDSF-UKQCD Collaboration), A lattice study of the glue in the nucleon, Phys. Lett. B 714, 312 (2012).

[34] X. Ji, Parton Physics on a Euclidean Lattice, Phys. Rev. Lett. 110, 262002 (2013).

[35] Y. Q. Ma and J. W. Qiu, Extracting parton distribution functions from lattice QCD calculations, Phys. Rev. D 98, 074021 (2018).

[36] A. V. Radyushkin, Quasi-parton distribution functions, momentum distributions, and pseudo-parton distribution functions, Phys. Rev. D 96, 034025 (2017).

[37] Y. Q. Ma and J. W. Qiu, Exploring Partonic Structure of Hadrons Using ab initio Lattice QCD Calculations, Phys. Rev. Lett. 120, 022003 (2018).

[38] A. J. Chambers, R. Horsley, Y. Nakamura, H. Perlt, P. E. L. Rakow, G. Schierholz, A. Schiller, K. Somfleth, R. D. Young, and J. M. Zanotti (QCDSF Collaboration), Nucleon Structure Functions from Operator Product Expansion on the Lattice, Phys. Rev. Lett. 118, 242001 (2017).

[39] K. Orginos, A. Radyushkin, J. Karpie, and S. Zafeiropoulos, Lattice QCD exploration of parton pseudo-distribution functions, Phys. Rev. D 96, 094503 (2017).

[40] C. Alexandrou, K. Cichy, M. Constantinou, K. Jansen, A. Scapellato, and F. Steffens, Light-Cone Parton Distribution
Functions from Lattice QCD, Phys. Rev. Lett. 121, 112001 (2018).

[41] G. S. Bali, V. M. Braun, B. Gläßle, M. Göckeler, M. Gruber, F. Hutzler, P. Korcyl, A. Schäfer, P. Wein, and J.-H. Zhang, Pion distribution amplitude from Euclidean correlation functions: Exploring universality and higher-twist effects, Phys. Rev. D 98, 094507 (2018).

[42] H. W. Lin, J.-W. Chen, X. Ji, L. Jin, R. Li, Yu.-S. Liu, Y.-B. Yang, J.-H. Zhang, and Y. Zhao, Proton Isovector Helicity Distribution on the Lattice at Physical Pion Mass, Phys. Rev. Lett. 121, 242003 (2018).

[43] R. S. Sufian, J. Karpie, C. Egerer, K. Orginos, J. W. Qiu, and D. G. Richards, Pion valence quark distribution from matrix element calculated in lattice QCD, Phys. Rev. D 99, 074507 (2019).

[44] G. S. Bali et al. (RQCD Collaboration), Light-cone distribution amplitudes of octet baryons from lattice QCD, Eur. Phys. J. A 55, 116 (2019).

[45] T. Izubuchi, L. Jin, C. Kallidonis, N. Karthik, S. Mukherjee, P. Petreczky, C. Shugert, and S. Syritsyn, Valence parton distribution function of pion from fine lattice, Phys. Rev. D 100, 034516 (2019).

[46] J. Liang, T. Draper, K.-F. Liu, A. Rothkopf, and Y.-B. Yang ( $\chi$ QCD Collaboration), Towards the nucleon hadronic tensor from lattice QCD, Phys. Rev. D 101, 114503 (2020).

[47] B. Joó, J. Karpie, K. Orginos, A. Radyushkin, D. Richards, and S. Zafeiropoulos, Parton distribution functions from Ioffe time pseudo-distributions, J. High Energy Phys. 12 (2019) 081.

[48] K. Cichy and M. Constantinou, A guide to light-cone PDFs from lattice QCD: An overview of approaches, techniques and results, Adv. High Energy Phys. 2019, 3036904 (2019).

[49] B. Yoon, Y. C. Jang, R. Gupta, T. Bhattacharya, J. Green, B. Jo, H. W. Lin, K. Orginos, D. Richards, S. Syritsyn, and F. Winter, Isovector charges of the nucleon from $2+1$-flavor QCD with clover fermions, Phys. Rev. D 95, 074508 (2017).

[50] B. L. Ioffe, Space-time picture of photon and neutrino scattering and electroproduction cross-section asymptotics, Phys. Lett. 30B, 123 (1969).

[51] W. Morris, Y. Q. Ma, J. W. Qiu, and A. V. Radyushkin (to be published).

[52] G. F. Sterman, Summation of large corrections to short distance hadronic cross-sections, Nucl. Phys. B281, 310 (1987).

[53] S. Catani and L. Trentadue, Resummation of the QCD perturbative series for hard processes, Nucl. Phys. B327, 323 (1989).

[54] H. Shimizu, G. F. Sterman, W. Vogelsang, and H. Yokoya, Dilepton production near partonic threshold in transversely polarized proton-antiproton collisions, Phys. Rev. D 71, 114007 (2005).

[55] J. C. Collins, D. E. Soper, and G. F. Sterman, Transverse momentum distribution in Drell-Yan pair and $\mathrm{W}$ and $\mathrm{Z}$ boson production, Nucl. Phys. B250, 199 (1985).

[56] Z. Y. Li, Y. Q. Ma, and J. W. Qiu, Extraction of next-to-nextto-leading-order PDFs from lattice QCD calculations, arXiv:2006.12370. 
[57] R. Edwards, B. Joó, K. Orginos, D. Richards, and F. Winter, U.S. $2+1$ flavor clover lattice generation program (2016) (to be published).

[58] C. G. Boyd, B. Grinstein, and R. F. Lebed, Constraints on Form-Factors for Exclusive Semileptonic Heavy to Light Meson Decays, Phys. Rev. Lett. 74, 4603 (1995).

[59] C. Bourrely, I. Caprini, and L. Lellouch, Model-independent description of $B \rightarrow \pi l \nu$ decays and a determination of $|V(u b)|$, Phys. Rev. D 79, 013008 (2009); Erratum, Phys. Rev. D 82, 099902 (2010).

[60] R. A. Briceño, J. V. Guerrero, M. T. Hansen, and C. J. Monahan, Finite-volume effects due to spatially nonlocal operators, Phys. Rev. D 98, 014511 (2018).

[61] B. Joó, J. Karpie, K. Orginos, A. V. Radyushkin, D. G. Richards, R. S. Sufian, and S. Zafeiropoulos, Pion valence structure from Ioffe time pseudo-distributions, Phys. Rev. D 100, 114512 (2019).

[62] V. N. Gribov and L. N. Lipatov, Deep inelastic e p scattering in perturbation theory, Yad. Fiz. 15, 781 (1972) [Sov. J. Nucl. Phys. 15, 438 (1972)].

[63] G. Altarelli and G. Parisi, Asymptotic freedom in parton language, Nucl. Phys. B126, 298 (1977).

[64] Y. L. Dokshitzer, Calculation of the structure functions for deep inelastic scattering and e+ e- annihilation by perturbation theory in quantum chromodynamics, Sov. Phys. JETP 46, 641 (1977) [Zh. Eksp. Teor. Fiz. 73, 1216 (1977)].

[65] R. Brun and F. Rademakers, ROOT-An object oriented data analysis framework, Nucl. Instrum. Methods Phys. Res., Sect. A 389, 81 (1997).

[66] A. Buckley, J. Ferrando, S. Lloyd, K. Nordström, B. Page, M. Rüfenacht, M. Schönherr, and G. Watt, LHAPDF6: parton density access in the LHC precision era, Eur. Phys. J. C 75, 132 (2015).
[67] J. Karpie, K. Orginos, A. Rothkopf, and S. Zafeiropoulos, Reconstructing parton distribution functions from Ioffe time data: From Bayesian methods to neural networks, J. High Energy Phys. 04 (2019).

[68] T. Regge, Introduction to complex orbital momenta, Nuovo Cimento 14, 951 (1959).

[69] S. J. Brodsky and G. R. Farrar, Scaling Laws at Large Transverse Momentum, Phys. Rev. Lett. 31, 1153 (1973).

[70] J. H. Zhang, J. W. Chen, L. Jin, H. W. Lin, A. Schäfer, and Y. Zhao, First direct lattice-QCD calculation of the $x$-dependence of the pion parton distribution function, Phys. Rev. D 100, 034505 (2019).

[71] L. Chang, C. Mezrag, H. Moutarde, C. D. Roberts, J. Rodrguez-Quintero, and P. C. Tandy, Basic features of the pion valence-quark distribution function, Phys. Lett. B 737, 23 (2014).

[72] J. Towns et al., XSEDE: Accelerating scientific discovery, Comput. Sci. Eng. 16, 62 (2014).

[73] R. G. Edwards and B. Joó (SciDAC, LHPC, and UKQCD Collaborations), The chroma software system for lattice QCD, Nucl. Phys. B, Proc. Suppl. 140, 832 (2005).

[74] M. A. Clark, R. Babich, K. Barros, R. C. Brower, and C. Rebbi, Solving lattice QCD systems of equations using mixed precision solvers on GPUs, Comput. Phys. Commun. 181, 1517 (2010).

[75] R. Babich, M. A. Clark, and B. Joo, Parallelizing the QUDA library for multi-GPU calculations in lattice quantum chromodynamics, arXiv:1011.0024.

[76] B. Joó, D. D. Kalamkar, T. Kurth, K. Vaidyanathan, and A. Walden, Optimizing Wilson-Dirac operator and linear solvers for Intel KNL, in High Performance Computing. ISC High Performance 2016, Lecture Notes in Computer Science Vol. 9945 (Springer, Cham, 2016). 